Vol. 18, n² | 2014

Varia

\title{
Dominique Kalifa, Pierre Karila-Cohen (dir.), Le commissaire de police au XIX ${ }^{e}$ siècle
}

\section{Antoine Renglet}

\section{(2) OpenEdition}

\section{Journals}

Édition électronique

URL : http://journals.openedition.org/chs/1502

DOI : $10.4000 /$ chs. 1502

ISSN : 1663-4837

Éditeur

Librairie Droz

\section{Édition imprimée}

Date de publication : 1 octobre 2014

Pagination : 126-128

ISBN : 978-2-600-01854-8

ISSN : $1422-0857$

\section{Référence électronique}

Antoine Renglet, " Dominique Kalifa, Pierre Karila-Cohen (dir.), Le commissaire de police au XIXe siècle », Crime, Histoire \& Sociétés / Crime, History \& Societies [En ligne], Vol. 18, n² | 2014, mis en ligne le 01 octobre 2014, consulté le 22 septembre 2020. URL : http://journals.openedition.org/chs/1502 ; DOI : https://doi.org/10.4000/chs. 1502

Ce document a été généré automatiquement le 22 septembre 2020.

(c) Droz 


\title{
Dominique Kalifa, Pierre Karila- Cohen (dir.), Le commissaire de police au XIX siècle
}

\author{
Antoine Renglet
}

\section{RÉFÉRENCE}

Dominique Kalifa, Pierre Karila-Cohen (dir.), Le commissaire de police au XIX ${ }^{e}$ siècle, Paris, Publications de la Sorbonne, 2008, 284 pp., ISBN 9782859445959.

Depuis plusieurs années, grâce aux travaux de Dominique Monjardet ${ }^{1}$ rapprochant $^{2}$ police et monde du travail, l'histoire de la professionnalisation policière ${ }^{2}$ connait un intérêt grandissant. Dans ce renouveau historiographique, la figure du commissaire de police a, jusque récemment, peu retenu l'attention des chercheurs ${ }^{3}$. L'ouvrage collectif dirigé par Dominique Kalifa et Pierre Karila-Cohen, édité aux Publications de la Sorbonne, vient pallier cette lacune en rassemblant une dizaine de contributions consacrées à cette fonction et aux hommes qui l'ont exercée dans la France d'un XIX ${ }^{\mathrm{e}}$ siècle qui s'ouvre à la période révolutionnaire et se termine à la Première Guerre mondiale. S'y ajoute un élargissement par une comparaison avec la fonction de police superintendent anglais et par une mise en perspective sociologique. En grands spécialistes de la France du XIX ${ }^{\mathrm{e}}$ siècle et de l'histoire de la justice à cette époque, les deux directeurs de ce livre introduisent celui-ci par une remise en contexte législative, historiographique et culturelle. L'ambition de l'ouvrage est clairement indiquée: il s'agit d'approcher les représentations actuelles et passées ainsi que les origines de cet «homme de l'entre-deux » qu'est le commissaire de police - institution à mi-chemin entre l'État et la société, entre les pouvoirs publics et la rue, entre les élites et le peuple - et qui demeure une pièce maîtresse du paysage institutionnel français. L'ouvrage s'organise en quatre volets successifs. L'ensemble est suivi de plusieurs annexes et d'une bibliographie. 
2 La première partie retrace les évolutions du profil socioprofessionnel des commissaires. Dans sa contribution consacrée aux commissaires de police parisiens sous la Révolution, Vincent Denis décrit la progressive stabilisation d'une institution qui se reconstruit sur les ruines de son ancêtre d'Ancien Régime. Élus entre 1790 et 1795, les commissaires, sous le Directoire, sont nommés par l'administration départementale sur proposition de la municipalité. Après brumaire de l'an VIII, la centralisation est renforcée puisque c'est le ministre de la Police puis l'Empereur lui-même, qui les nomme. Il résulte de cette variation du procédé de sélection un groupe professionnel en constante évolution. Jean-Paul Jourdan propose quant à lui de comparer le profil social des commissaires de police alsaciens, étudié par Édouard Ebel ${ }^{4}$, avec celui des Aquitains entre 1800 et 1870 . Il montre dans une première partie consacrée à leur origine géographique la part grandissante, au cours du XIX ${ }^{e}$ siècle, de commissaires allochtones de même que leur origine urbaine presque généralisée. Dans un deuxième temps, Jean-Paul Jourdan met en évidence la proportion importante d'anciens militaires dans les rangs policiers. Il aborde également la question de la rétribution des commissaires, de leur fortune et de leur notabilité. Pour la même période, Alexandre Nugues-Bourchat analyse de manière précise pour la ville de Lyon, la question, généralement peu évoquée par les chercheurs - y compris dans cet ouvrage -, des rapports entre les commissaires et la population. Le commissaire de police apparaît comme l'acteur essentiel du maintien de l'ordre mais aussi comme le relais entre les gouvernants et les gouvernés. Conscients de ce statut, les commissaires cherchent autant à faire valoir à leur profession une notabilité depuis longtemps acquise par leurs homologues de la justice qu'à remplir les multiples tâches du quotidien qui leur incombent: accueil de la population, rédaction de procès-verbaux, correspondance avec leurs supérieurs, etc. Dialogue, proximité, souplesse et interprétation de la loi semblent dès lors qualifier le travail des commissaires à Lyon au XIX ${ }^{e}$ siècle.

3 La deuxième partie, consacrée à la construction progressive du métier de policier au cours de ce long siècle, s'ouvre sur une contribution de Pierre Karila-Cohen qui s'interroge sur la période de «crise» de l'institution sous la monarchie constitutionnelle. L'analyse d'une dizaine de mémoires de policiers de la Restauration montre en effet que la fonction de commissaire de police connaît à cette époque une crise profonde révélée par l'absence de considération et la précarité dont les policiers affirment faire l'objet. En analysant les révocations au cours de cette même période, John Merriman montre que l'incompétence, la négligence et la désobéissance des commissaires sont évoquées de manière croissante comme raison de destitution, au contraire des sympathies politiques pointées au cours des régimes précédents. Au nombre des motifs de plus en plus invoqués et qui traduisent une professionnalisation accrue de la fonction, il faut ajouter l'alcoolisme, le grand âge et la mauvaise fortune financière des commissaires de police. La reprise en main des polices municipales par l'État se concrétise sous le Second Empire. En centrant son analyse sur le Puy-de-Dôme, Cyril Cartayrade explique que cette période est marquée par un renforcement de la présence gendarmique dans les campagnes au détriment des commissariats. Laurent López montre également que les rapports entre commissaires de police et officiers de gendarmerie, s'ils sont parfois entachés de conflits ou de règlements de comptes interpersonnels, permettent le plus souvent une collaboration efficace. La revalorisation de l'image des commissaires y est manifestement pour beaucoup. Sous la Troisième République en effet, le rapprochement entre les deux corps s'accentue et la professionnalisation des commissaires de police s'impose. 
4 La troisième partie de l'ouvrage met en parallèle deux itinéraires de commissaires. Jean-François Tanguy analyse finement les conflits qui ont jalonné la carrière d'Eugène Court, ex-coiffeur toulousain devenu commissaire central à Rennes sous la $\mathrm{III}^{\mathrm{e}}$ République. La fonction qu'il incarne a pour mission de s'opposer à l'action de la municipalité en matière de police et ainsi de permettre un renforcement de la centralisation et du contrôle politique. Jean-Marc Berlière s'attache de son côté à retracer la carrière exceptionnelle de Célestin Hennion, modeste inspecteur spécial de deuxième classe, qui parvient à se hisser, mission après mission, au poste de directeur de la Sûreté générale puis à celui de Préfet de police. Sa carrière culmine sous l'ère Clemenceau qui le charge de mettre en place les brigades mobiles de police judiciaire, les célèbres brigades du Tigre, véritable modèle incarné d'une police républicaine.

Enfin, dans la quatrième partie, deux élargissements sont proposés. Philippe Chassaigne compare ainsi, pour le XIX ${ }^{\mathrm{e}}$ siècle, la figure du police superintendent anglais au commissaire de police français. Il pointe tout d'abord la faible attention dont cet officier de police a fait l'objet par les historiens, contrairement au populaire police constable, pourtant son subalterne. Ensuite, il montre que la caractéristique du commissaire de police anglais est d'être avant tout un maillon intermédiaire entre les rangs inférieurs et supérieurs de la police anglaise. Même si sa présence est obligatoire au sein de sa division et qu'il est tenu à des inspections, le superintendent est davantage un superviseur de tâches et un relais administratif qu'un agent de terrain au contact de la population. Dans le deuxième élargissement, Frédéric Ocqueteau pose la question des liens entre le commissaire de police du XIX siècle et celui du XXI ${ }^{e}$ siècle. Il revient sur chacun des textes de l'ouvrage en pointant, ici et là, ce qui peut surprendre le sociologue ou l'inviter à établir des comparaisons avec le passé. Frédéric Ocqueteau décrit également la fracture que représente l'étatisation de la police en 1941 et l'octroi, avec la loi du 28 septembre 1948, d'un «statut spécial " à l'institution, statut dérogatoire au droit commun. Enfin, il aborde la question de la mobilité fonctionnelle et de la mobilité géographique dans les trajectoires de carrière des commissaires d'aujourd'hui.

6 Au terme de cet aperçu sommaire, il convient de conclure que, même s'il n'est pas une synthèse, l'ouvrage de Dominique Kalifa et de Pierre Karila-Cohen fait utilement le point sur les connaissances actuelles de la profession de commissaire de police au XIX ${ }^{\mathrm{e}}$ siècle en réunissant les contributions des meilleurs spécialistes du sujet. Pour cette raison, il sera grandement apprécié par tous ceux qui s'intéressent à des thématiques touchant de près ou de loin à ces questions. Osons toutefois formuler ce regret: les rapports entre les commissaires et la population, de même que leur travail quotidien, ne restent généralement abordés qu'en filigrane des recherches présentées.

\section{NOTES}

1. Monjardet Dominique, Ce que fait la police. Sociologie de la force publique, Paris, La Découverte, 1996. 
2. Berlière Jean-Marc et al. (dir.), Métiers de police. Être policier en Europe, XVIII ${ }^{e}-X X^{e}$ siècles, Rennes, Presses universitaires de Rennes, 2008.

3. En mai 2012, les commissaires de police sous la Révolution et l'Empire ont fait l'objet d'une journée d'étude organisée à l'Université de Namur (Belgique).

4. Ebel Édouard, Police et société. Histoire de la police et de son activité en Alsace au XIXe siècle, Strasbourg, Presses universitaires de Strasbourg, 1999.

\section{RÉSUMÉS}

Paris, Publications de la Sorbonne, 2008, 284 pp., ISBN 9782859445959

\section{AUTEURS}

\section{ANTOINE RENGLET}

Université de Namur/Université de Lille-3 